\title{
Wohlwollendes Anschubsen: Was ist mit liberalem Paternalismus zu erreichen und was sind seine Nebenwirkungen?
}

\author{
Jan Schnellenbach*
}

Walter-Eucken-Institut, Freiburg i. Br. und Ruprecht-Karls-Universität Heidelberg

\section{Einleitung}

Die empirische Verhaltensökonomik hat sowohl in experimentellen, als auch in Feldstudien in den vergangenen Jahren beeindruckende und überzeugende Evidenz zusammengetragen, die zeigt, dass Menschen sehr wahrscheinlich nicht durch eine vollständige, in der Zeit konstante und widerspruchsfreie Präferenzordnung zu charakterisieren sind. Damit wird nicht nur ein Grundpfeiler der neoklassischen Lehrbuch-Mikroökonomik infrage gestellt, sondern auch die ganz praktische Vorstellung, dass jeder Mensch im Zweifelsfall selbst am besten weiß, was sein eigenes Wohlergehen maximiert. Denn wenn Präferenzen inkonsistent sind, dann ist das morgige Bereuen des Konsums von heute unter Umständen nicht nur auf einen zufälligen Fehler zurückzuführen, sondern auf ein tiefer liegendes und systematisches Problem.

Solche Entscheidungsanomalien haben im Fahrwasser der Verhaltensökonomik, und verfasst von einigen prominenten Verhaltensökonomen, das Konzept des liberalen Paternalismus entstehen lassen. Es geht hier um die Frage, wie man Individuen dabei helfen kann Entscheidungen $\mathrm{zu}$ treffen, die für sie selbst besser sind als solche Entscheidungen, die sie ohne paternalistische Hilfe treffen würden. Im Zentrum steht dabei der Versuch, die Randbedingungen von Entscheidungssituationen im Hinblick auf eine Verbesserung der Entscheidungsqualität zu gestalten. Liberaler Paternalismus beschäftigt sich also ausdrücklich nicht mit Externalitäten oder ähnlichen klassischen Motiven für die Korrektur individueller Entscheidungen, sondern ausschließlich mit der Förderung des individuellen Wohlergehens des Entscheidungsträgers selbst.

In diesem kurzen Papier soll nun kritisch untersucht werden, ob liberaler Paternalismus tatsächlich ein taugliches Konzept zur Verbesserung individueller Entscheidungen ist. In Abschnitt 2 wird das Konzept selbst kurz erläutert, in Abschnitt 3 erfolgt ein Überblick über seine verhaltensökonomischen Grundlagen. In Abschnitt 4 erfolgt die Diskussion einiger theoretischer und politischökonomischer Probleme des liberalen Paternalismus und Abschnitt 5 schließt die Argumentation mit einem Fazit ab.

\footnotetext{
*Kontaktadresse: Walter-Eucken-Institut, Goethestr. 10, 79100 Freiburg im Breisgau, E-mail: schnellenbach@walter-eucken-institut.de.
} 


\section{Wie funktioniert liberaler Paternalismus?}

Die Grundidee des liberalen Paternalismus wird bereits im Titel des Bestsellers von Richard Thaler und Cass Sunstein (2008) formuliert, mit dem das Konzept popularisiert wurde: Nudge. Es geht um das Anschubsen der Individuen mit dem Ziel, ihnen das Fällen guter Entscheidungen zu erleichtern. Was genau wir dabei unter einer guten Entscheidung zu verstehen haben, werden wir noch in der folgenden Diskussion zu klären haben. Wichtig ist zunächst, dass liberale Paternalisten erklären, ihren Mitmenschen keine Vorschriften machen zu wollen, welche durch harte Sanktionen, wie etwa durch Strafandrohungen, durchzusetzen wären. Entsprechend spricht man gelegentlich anstelle von liberalem auch synonym von weichem Paternalismus.

Wenn man etwa glaubt, dass die Individuen ihren Alkoholkonsum nicht rational planen können, weil sie negative gesundheitliche Folgen systematisch unterschätzen, dann würde die typische Reaktion eines traditionellen Paternalisten auf diesen Sachverhalt in der Forderung nach einer völligen Alkoholprohibition bestehen, oder auch in der Einführung von hohen Konsumsteuern auf alkoholische Getränke. Wenn ein Konsument über die negativen Folgen des Alkoholkonsums unzureichend informiert ist, dann kann ihn immerhin das Preissignal zu einer rationaleren Entscheidung bewegen. Der weiche Paternalist hingegen würde beim Design von Entscheidungssituationen ansetzen. Er würde die Spirituosen in die hinteren Ecken der Geschäfte verbannen und dadurch Impulskäufe einschränken, wohlüberlegte Konsumentscheidungen aber nicht wirklich behindern (Sunstein und Thaler 2003). Oder er würde für das Anbringen gut sichtbarer und möglichst abschreckender Etiketten plädieren, die den Konsumenten über die negativen Langfristfolgen seines Alkoholkonsums im Moment der Kaufentscheidung informieren.

Die Beispiele für weichen Paternalismus sind inzwischen in der Literatur recht zahlreich. So diagnostizieren etwa Thaler und Benartzi (2004) unter den für ihre Altersvorsorge sparenden Arbeitnehmern erhebliche Selbstkontrollprobleme, sowie eine Neigung zum Aufschieben: Auch solche Studienteilnehmer, die sich selbst eine zu niedrige Sparquote attestieren, sind demnach nur schwer dazu zu bewegen, tatsächlich auf zusätzlichen Gegenwartskonsum zu verzichten. Dies ändert sich mit einem Vertrag, in dem die Studienteilnehmer sich verbindlich verpflichten, in der Zukunft ihre Sparquote anzuheben. Diese Selbstverpflichtung wird tatsächlich von einem großen Teil der Individuen auch durchgehalten; dies deutet darauf hin, dass es eine freiwillige Nachfrage nach einem solchen Mechanismus zur Lösung eines spezifischen Selbstkontrollproblems gibt.

Eine andere Variante des weichen Paternalismus besteht in einer Änderung der Rückfallposition in einer bestimmten Entscheidungssituation. Thaler und Sunstein (2008) berichten etwa von einer Highschool im Bundesstaat Texas, die von allen Schülern fordert, sich vor der Zeugnisausgabe im örtlichen Community College einzuschreiben. Natürlich steht es jedem Absolventen frei, sich anschließend für eine qualitativ bessere Hochschule zu entscheiden, oder ganz auf eine akademische Ausbildung zu verzichten. Thaler und Sunstein begrüßen dennoch ausdrücklich das Resultat, dass durch die Zwangseinschreibung die Quote der Schüler ansteigt, die schlussendlich auch tertiäre Bildung genießen.

Die aus Sicht des etablierten Konsumentenschutzes sicherlich konventionellste Form des liberalen Paternalismus betrifft die Bereitstellung von Informationen für 
den Konsumenten (z.B. Camerer et al. 2003, S. 1232ff.). Jedoch muss man hier genau unterscheiden, in welcher Weise Verpflichtungen zur umfassenden Information von Konsumenten definiert werden. So dürfte beispielsweise eine Pflicht zur bloßen Aufzählung von Inhaltsstoffen von Lebensmitteln relativ unproblematisch sein. Für den Konsumenten werden Informationskosten gesenkt, während die Wahrscheinlichkeit einer gezielten Manipulation seiner Wahlentscheidung eher gering ist. Auf der anderen Seite haben gerade Verhaltensökonomen immer wieder auf die Bedeutung von Framing-Effekten hingewiesen, d.h. auf die Möglichkeit, durch eine geschickte Aufbereitung von Informationen die Entscheidungen der Individuen in eine gewünschte Richtung zu lenken (Tversky und Kahneman 1981). Auch wenn es schwierig ist, eine exakte und allgemeingültige Grenze zwischen Konsumentenschutz und paternalistischer Intervention anzugeben, so ist bei einer gezielten Ausnutzung von psychologischen Framing-Effekten sicherlich die Grenze zum Paternalismus überschritten. Beispiele hierfür sind die Abbildung von Raucherlungen auf Zigarettenpackungen, ebenso wie eine besonders stark die Risiken betonende Beratung bei Finanzgeschäften, oder auch der Vorschlag der Lebensmittelampel in Ergänzung einer neutralen Aufzählung von Inhaltsstoffen und Nährwerten.

In jedem Fall bezieht sich liberaler Paternalismus also auf den Versuch, Entscheidungssituationen bewusst $\mathrm{zu}$ gestalten und so Individuen in ihrem Handeln zu lenken. Ein wichtiges Argument ist, dass die Details solcher Situationen bisher meist zufällig zustande kommen, aber jedenfalls nicht in einer Weise gestaltet sind, die das Wohlergehen des Entscheidungsträgers selbst zum Ziel hat. Ihre zentrale Aufgabe sehen liberale Paternalisten nun darin, die Randbedingungen von Entscheidungen systematisch so zu gestalten, dass ein Anschubsen in die von ihnen als richtig erkannte Richtung erfolgt, zugleich aber die Freiheit erhalten bleibt, bewusst eine abweichende Entscheidung zu treffen. ${ }^{1}$ Im folgenden Abschnitt erfolgt ein kurzer Überblick über wesentliche verhaltensökonomische Einsichten, auf denen der liberale Paternalismus aufbaut.

\section{Verhaltensökonomische Grundlagen des liberalen Paternalismus}

Die jüngere theoretische Verhaltensökonomik ist von zahlreichen empirischen Resultaten motiviert, die darauf hindeuten, dass Menschen sich häufig nicht so verhalten, wie man es von homines oeconomici erwarten würde. Für eine Diskussion des liberalen Paternalismus sind hier vor allem solche Ansätze interessant, die zu verstehen helfen, wieso Individuen gelegentlich Entscheidungen treffen, die ihrem Eigeninteresse widersprechen, oder zumindest aus der Perspektive eines neutralen Beobachters zu widersprechen scheinen.

Im oben bereits erwähnten Fall der Altersvorsorge etwa zeigt sich eine Diskrepanz zwischen den Absichtserklärungen der Individuen und ihrem tatsächlichen Sparverhalten (Choi et al. 2006). Hinzu kommt ein weiteres Faktum: Gegeben einen abnehmenden Grenznutzen des Konsums würde man erwarten, dass die Haushalte ihren Konsum über die Zeit glätten; stattdessen beobachtet man regelmäßig, wie

1. Weitere grundlegende Beiträge zum liberalen (oder weichen) Paternalismus sind Camerer et al. (2003) sowie zur Frage der wohlfahrtstheoretischen Fundierung paternalistischer Interventionen Loewenstein und Ubel (2008). 
beim Ausscheiden aus dem aktiven Arbeitsleben das Konsumniveau stark sinkt (Bernheim und Rangel 2007). Beide Beobachtungen zusammen führen zu der Vermutung, dass die Individuen insofern irrational handeln, als dass sie sich nicht ihren eigenen, langfristigen Präferenzen entsprechend verhalten. Hierfür werden dann in der Literatur kognitive Defizite oder Bildungsmängel verantwortlich gemacht, die den Individuen eine langfristige Planung verunmöglichen, oder auch Probleme der Selbstkontrolle, die mit der Annahme quasi-hyperbolischer Diskontierung modelliert werden (Laibson 1997). ${ }^{2}$

Ein ähnliches Argument lässt sich für das Ernährungsverhalten konstruieren. Brunello et al. (2009) legen Evidenz für die negativen Auswirkungen von erheblichem Übergewicht vor. Die Individuen müssen mit negativen Produktivitätsund Einkommenseffekten als Folge von Gesundheitsproblemen rechnen. Ihre geäußerten langfristigen Präferenzen deuten darauf hin, dass sie sich dessen durchaus bewusst sind. Sie handeln aber, als hätten sie zeitinkonsistente Präferenzen: Wenn sie nur den nächsten marginalen Snack im Auge haben, so unterschätzen sie dessen negativen langfristigen Effekt und greifen zu. Gruber und Köszegi (2004) argumentieren ähnlich für das Problem des Zigarettenrauchens und raten zur Nikotinbesteuerung, um den Anreiz zur Selbstkontrolle zu erhöhen.

Ebenso ist es denkbar, dass Individuen absichtlich relevante Information ausblenden. Akerlof und Dickens (1982) argumentieren zum Beispiel, dass Arbeitnehmer mit besonders gefährlichen Arbeitsplätzen zur Vermeidung psychischer Kosten diese Risiken ausblenden und folglich auch Sicherheitsausrüstungen in geringerem Maße nachfragen, als es eigentlich rational wäre. Einen interessanten Ansatz formulieren schließlich auch Cordes und Schubert (2011). In ihrem Modell können Konsumenten ihre Präferenzen verändern, indem sie von Vorbildern lernen. Eine solche Präferenzdynamik kann unter ungünstigen Bedingungen so verlaufen, dass die Individuen systematisch unerfüllbare Wünsche hegen und entsprechend unglücklich sind. Dies führt die Autoren zu dem Vorschlag, dass der Staat zwar nicht regulierend, aber doch im Sinne eines weichen Paternalismus informierend und Statusrennen abmildernd eingreifen solle.

Dieser kurze und keinesfalls vollständige Überblick zeigt, dass die Fälle zahlreich sind, in denen individuelles Handeln und ein vermutetes langfristiges Eigeninteresse nicht notwendig übereinstimmen. Wenn dies so ist, dann erscheint die Hoffnung der liberalen Paternalisten zunächst einmal plausibel, dass es

2. Diese Form der Diskontierung führt zu zeitinkonsistenten Präferenzen. Individuen werden dann nicht einen einmal für einen längeren Zeitraum aufgestellten Konsumplan einfach abarbeiten, wie es im Fall von Standardpräferenzen mit exponentiellem Diskontieren der Fall wäre. Vielmehr existiert jeweils in der Gegenwart zusätzlich zum exponentiellen Diskontieren eine weitere Verzerrung hin zum Gegenwartskonsum, die dazu führt, dass die Zukunft schwächer gewichtet wird. Dies wiederum führt dazu, dass die Individuen sich systematisch selbst enttäuschen: Pläne, die aus der längeren Vorausschau sinnvoll erscheinen, werden verworfen, wenn es ernst wird. So kann es beispielsweise aus der heutigen Sicht vorteilhaft erscheinen, in einem halben Jahr mit dem Sparen für eine in einem Jahr geplante Weltreise zu beginnen. Wenn es dann in einem halben Jahr soweit ist, kann es bei hyperbolischem Diskontieren aber dazu kommen, dass die erneute Gegenwartsorientierung dazu führt, dass der Sparplan aufgegeben wird. Individuen, die dies voraussehen, könnten aber bereits zu Beginn versuchen, sich beispielsweise durch entsprechende Verträge selbst an ihren ursprünglichen Plan zu binden und so die spätere Selbst-Enttäuschung zu verhindern. 
Möglichkeiten zu Wohlfahrtsverbesserungen der betroffenen Individuen ${ }^{3}$ geben müsse. Im folgenden Abschnitt wird im Detail diskutiert, inwiefern das tatsächlich der Fall ist und wo die Probleme des neuen Paternalismus liegen.

\section{Liberaler Paternalismus als effiziente Planung individueller Entscheidungssituationen: eine kritische Diskussion}

\subsection{Liberaler versus harter Paternalismus?}

Bei genauem Hinsehen erscheint die von den Vertretern des liberalen Paternalismus propagierte Neuheit ihres Ansatzes gegenüber dem traditionellen, harten Paternalismus nur als graduell, aber nicht als prinzipiell. Ein Unterschied zwischen beiden Konzepten wird mit dem Argument begründet, dass das Design von Wahlentscheidungen, wie es der neue Paternalismus fordert, den Individuen ihre Wahlfreiheit weiterhin gewähre. Traditionellere Interventionen dagegen seien darauf angelegt, unmittelbar die Menge der zur Wahl stehenden Alternativen $\mathrm{zu}$ reduzieren; sie seien also mit einer echten Einschränkung der Wahlfreiheit verbunden.

Die Erfahrung spricht jedoch nicht dafür, dass letzteres tatsächlich der Fall ist. Ein Beispiel kann dies veranschaulichen: Die Prohibition, die zwischen 1920 und 1933 den Alkoholkonsum in den USA durch eine Verfassungsnorm untersagte, kann sicherlich als Beispiel für harten Paternalismus gelten. Die Herstellung und der Verkauf alkoholischer Getränke wurden unter hohe Strafen gestellt. Die Prohibition schützte jedoch gerade die schweren Trinker wohl nicht umfassend vor sich selbst, denn die Anzahl der Fälle von Leberzirrhose sank infolge dieses traditionell paternalistischen Eingriffs nur um 10-20\% (Dillis und Miron 2004). Der traditionelle paternalistische Eingriff reduziert nicht die Anzahl der Alternativen, er verändert lediglich die relativen Preise. Alles Weitere ist dann eine Frage der Preiselastizität der Nachfrage nach dem betroffenen Konsumgut. Nüchtern ökonomisch betrachtet gilt also, dass die Sanktionen, mit denen harter Paternalismus Entscheidungen zu lenken versucht, nichts anderes als Preise sind. ${ }^{4}$

Genau eine solche Veränderung relativer Preise leistet aber auch der weiche Paternalismus. Wer sich auf der Suche nach ungesunden Lebensmitteln nunmehr in die hinterste Ecke des Supermarktes begeben muss, der erfährt ebenfalls nichts anderes als eine Erhöhung des relativen Preises der von ihm präferierten Güter, und zwar durch eine Manipulation der Transaktionskosten. Der Unterschied zum harten Paternalismus besteht hier also aus ökonomischer Sicht - aus juristischer Sicht mag dies anders sein - in einem quantitativen, nicht aber in einem qualitativen Schritt. Damit sind aber auch die Grenzen zwischen beiden Konzepten letztendlich fließend.

Dies gilt auch für den anderen scheinbaren Unterschied zum traditionellen Paternalismus, der von liberalen Paternalisten angeführt wird: Die Behauptung, dass liberaler Paternalismus seinen Adressaten keine ihnen fremden Präferenzen

3. Diese müssen aber nicht unbedingt Pareto-Verbesserungen sein; eine Wohlfahrtssteigerung von Konsumenten wird beispielsweise häufig mit einem Verlust für die betroffenen Anbieter einhergehen.

4. Vgl. Gneezy und Rustichini (2000) mit ihrer schon im Titel ihres Papiers formulierten Einsicht: A fine is a price. Dort zeigt sich allerdings, dass die Einführung formaler negativer Sanktionen sogar gänzlich unerwartete Effekte haben und die Nachfrage nach der sanktionierten Handlung erhöhen kann. 
aufzwingen, sondern ihnen beim Umgang mit ihren eigenen, inkohärenten Präferenzen helfen will (etwa Camerer et al. 2003). Dies wiederum setzt allerdings eine Entscheidung auf Seiten des paternalistischen Planers voraus. Er muss wissen, in welche Richtung sein scheinbar wohlwollendes Anschubsen gehen soll, welchem Element der inkohärenten individuellen Präferenzordnung er also den Vorzug geben möchte. Angeleitet von verhaltensökonomischen Beiträgen, wie den in Abschnitt 3 diskutierten, wird dann häufig die theoretische Schablone eines Selbstkontrollproblems gewählt. Diese führt zu einer hohen Gewichtung langfristiger Präferenzen, sowie zu einem paternalistischen Design von Entscheidungssituationen, das die Individuen in Richtung eines mit diesen langfristigen Präferenzen konsistenten Handelns manipulieren soll.

Man sieht also, dass auch hier der paternalistische Planer eine Entscheidung für die Individuen trifft. Genauer gesagt wird im Einzelfall eine solche Entscheidung getroffen auf der Grundlage einer allgemeinen Vermutung, dass es sinnvoll sei langfristigen Präferenzen den Vorzug zu geben. Dies ist allerdings keineswegs zwingend, wie wir unten in Abschnitt 4.3 noch sehen werden. Vor allem haben wir es hier jedoch letztendlich mit der gleichen Art des vorgeblich wohlwollenden Entscheidens für andere $\mathrm{zu}$ tun, wie man sie im traditionellen Paternalismus auch beobachten kann. Der „alte“ Paternalist gab vor zu wissen, dass bestimmte Konsumgüter für die Individuen schädlich seien und diese daher vor solchen Gütern geschützt werden sollten; der „neue“ Paternalist gibt entsprechend vor zu wissen, welches Element einer widersprüchlichen Präferenzordnung unvernünftig und welches vernünftig ist.

Zwar ist es zweifellos ein Vorteil des liberalen Paternalismus, wenn die verhaltenssteuernde Manipulation relativer Preise nun geringer ausfällt, als es zuvor bei seinem Vorgänger der Fall war. Es wird Individuen mit hinreichend stark abweichenden Präferenzen leichter gemacht, sich ihrem eigentlich gewünschten Konsummuster anzunähern. Ein Problem bleibt aber dennoch gerade dann bestehen, wenn man aus verhaltensökonomischer Sicht die Vorstellung stabiler, gegebener Präferenzen ablehnt. Diejenigen Individuen, die (noch) keine stark abweichenden Präferenzen haben, werden durch paternalistisch-standardisierte Entscheidungssituationen der Möglichkeit beraubt, zufällig Neues zu entdecken und dadurch ihre Präferenzen in ex ante unerwartete, aber letztlich vielleicht ex post individuell wohlfahrtssteigernde Richtungen zu entwickeln. Die Behauptung der Harmlosigkeit des liberalen Paternalismus mit seinen relativ zum alten Paternalismus niedrigen Austrittsschwellen beruht also paradoxerweise auf einem sehr traditionellen, statischen und überhaupt nicht verhaltensökonomisch informierten Verständnis individueller Präferenzen.

\subsection{Ein Markt für Selbstbindungen}

In einer Hinsicht ist die Tatsache, dass Paternalismus nicht mehr als eine Veränderung relativer Preise bewirken kann, zunächst einmal bedauerlich. Denn Gul und Pesendorfer (2001) zeigen, dass Individuen zwar eine über die Zeit identische Präferenzordnung, aber dennoch auch eine Präferenz für Selbstbindung in der Zukunft haben können. ${ }^{5}$ Anders gesagt: Es kann passieren,

5. Ähnliche Überlegungen liegen in der klassisch-liberalen Sozialphilosophie der positiven Sichtweise zugrunde, die deren Autoren auf ein an Regeln, Konventionen und Institutionen orientiertes Handeln 
dass sogar homo oeconomicus eine Nachfrage nach Mechanismen äußert, die ihm die Selbstkontrolle erleichtern. Das völlige Verschwinden einer zukünftig unerwünschten Handlungsalternative aus der Menge der erreichbaren Alternativen wäre hierzu die sicherste Möglichkeit.

Dennoch gibt es für rationale, sich ihrer Präferenzen bewusste Individuen Mechanismen, Selbstbindungen freiwillig einzugehen. So berichten beispielsweise Kaur et al. (2010) von einem Arbeitsplatz-Experiment, in dem ein erheblicher Anteil der Teilnehmer freiwillig eine Vertragsvariante wählt, die eine geringere Entlohnung vorsieht für den Fall, dass ein zuvor vereinbartes Produktivitätsziel nicht erreicht wird. Da die Teilnehmer mit diesem Vertrag niemals mehr verdienen können als mit dem alternativen Vertragsarrangement, das eine fixe Entlohnung vorsieht, sind sie sich offenbar ihres Selbstkontrollproblems bewusst und suchen freiwillig ein zur Lösung dieses Problems geeignetes institutionelles Arrangement. Ähnliches funktioniert auch für andere Problembereiche, wie etwa die oben angesprochenen Sparentscheidungen (DellaVigna 2009). Der in Yale lehrende Ökonom Ian Ayres hat gar eine kommerzielle Internetplattform gegründet, auf der jedermann bindende Verträge abschliessen kann, die ihn zu einer hohen Geldzahlung verpflichten, falls er ein bestimmtes Ziel (z.B. einen Gewichtsverlust oder die Nikotinabstinenz) nicht bis zu einem vereinbarten Termin nachgewiesen hat. ${ }^{6}$

Wenn Individuen also langfristige Präferenzen haben, und wenn sie diese selbst so hoch gewichten, dass sie ihnen zum Durchbruch verhelfen wollen, dann finden sie sehr häufig Marktlösungen, die ihnen helfen, ihr Ziel zu erreichen. Solche dezentralen Lösungen haben Vorteile gegenüber Situationen, in denen von vornherein die Randbedingungen einer Entscheidung paternalistisch manipuliert wurden: Sie sind treffsicherer und sie werden individuell völlig freiwillig eingegangen. Somit ist auch die Vermutung plausibel, dass sie tatsächlich zu Wohlfahrtssteigerungen führen werden. Darüber hinaus lassen sie sich individuell maßschneidern - die Höhe der angedrohten Strafzahlung, die jemand zu seiner eigenen Selbstdisziplinierung für notwendig hält, wird er wohl am ehesten selbst abschätzen können. ${ }^{7}$

\subsection{Was maximieren liberale Paternalisten?}

Eine zentrale Frage ist, was eigentlich das Ziel liberaler Paternalisten ist. Dies ist keine triviale Frage, da die Vertreter dieses Ansatzes selbst die Existenz konsistenter individueller Präferenzen infrage stellen und unterstellen, dass jedes Individuum verschiedene Präferenzordnungen in sich vereint, die sich gegenseitig widersprechen können. Wenn man dieses Argument akzeptiert, dann sind aber auch die Grundlagen der etablierten Wohlfahrtsökonomik obsolet und ihre

haben (Wohlgemuth 2011). Auch ökonomisch modellierte Gewissensentscheidungen können als Ursache für eine Nachfrage nach Selbstbindungen gesehen werden (Heinemann 2010).

6. Vgl. The Economist, An Idea for Lent: Carrot and stickK. How to help your good self triumph over your bad self, Ausgabe vom 7. Februar 2008.

7. Eine Ausnahme ist allenfalls echtes Suchtverhalten. Bernheim und Rangel (2004) unterscheiden kontrollierte und unkontrollierte individuelle Zustände (cold and hot states) Abhängiger. In unkontrollierten Zuständen werden freiwillige Selbstbindungen nicht funktionieren, weicher Paternalismus allerdings ebenso wenig. 
Effizienzkonzepte nicht mehr ohne weiteres anwendbar. Es besteht also zunächst einmal ein Bedarf nach einem adäquaten Wohlfahrtsbegriff, der seinerseits in normativen Begründungen paternalistischer Interventionen Anwendung finden könnte. Für eine ausführliche Diskussion solcher alternativer Wohlfahrtskonzepte sei der Leser auf Cordes und Schubert (2011) verwiesen. Hier soll nur auf einzelne, für unsere Diskussion unmittelbar relevante Punkte eingegangen werden.

Sugden (2008) weist darauf hin, dass im Ansatz des liberalen Paternalismus die intervenierende Instanz idealtypisch die Rolle eines Schiedsrichters zwischen konfligierenden individuellen Präferenzen einnimmt. Liberale Paternalisten wollen erklärtermaßen den Individuen keine fremden Präferenzen aufzwingen, sondern ihnen lediglich bessere Entscheidungen bei gegebenen, wenn auch oft konfligierenden Präferenzen ermöglichen. Bei inkohärenten individuellen Präferenzen steht der paternalistischen Planer dann also vor der Entscheidung, welchen Komponenten der widersprüchlichen Präferenzordnungen er den Vorrang geben soll. Sunstein und Thaler (2003a) schlagen als normativen Maßstab die Handlungen eines Individuums vor, für die es sich entscheiden würde, wenn es mit unbegrenzten kognitiven Kapazitäten ausgestattet, von keinerlei Willensschwächen geplagt und vollständig informiert wäre. Es ist offensichtlich, dass dieser normative Maßstab utopisch und empirisch leer ist; hinzu käme im Fall der Anwendung dieses Maßstabes die Anmaßung von Wissen um die wahren Motive fremder Menschen.

Die paternalistische Praxis setzt also andere, leichter operationalisierbare Ziele voraus. Eine Möglichkeit, mit der Vertreter dieses Ansatzes immer wieder sympathisieren, besteht darin, langfristigen Präferenzen den Vorzug zu geben. Viele Menschen äußern den langfristigen Wunsch nach gesunder Ernährung, greifen aber dennoch kurzfristig immer wieder zur Eiscreme. Vor dem Hintergrund der oben diskutierten Ansätze ist es dann leicht, die Orientierung an der langfristigen Präferenzordnung als rational, aber das kurzfristig hiervon abweichende Handeln als zu überwindende Willensschwäche aufzufassen. Aber ist dies auch zwingend?

In seiner pragmatischen Anwendung ähnelt das Konzept des liberalen Paternalismus hochgradig der wesentlich älteren Idee der meritorischen Güter. Auch hier ging es darum, zunächst zu identifizieren, wann und wovon Individuen tendenziell ,zu wenig“ oder „zu viel“ konsumieren, um dann daraus Empfehlungen für staatliche Interventionen abzuleiten. Gründe für in diesem Sinne fehlerhafte individuelle Entscheidungen wurden etwa in falschen oder unvollständigen Informationen gesehen, aber auch darin, dass dem Konsum meritorischer Güter ein objektiver, von individuellen Nutzenschätzungen unabhängiger Wert beigemessen wurde, so dass ein Gut in der sozialen Wohlfahrtsfunktion mit einem anderen Gewicht als in den individuellen Nutzenfunktionen berücksichtigt wurde (Pazner 1972). Diese Differenz kann auch so gedeutet werden, dass die Individuen Präferenzen haben, die vom Standpunkt eines externen Beobachters aus beurteilt falsch sind (Besley 1988). Die Ähnlichkeit zum liberalen Paternalismus, der ebenfalls von einem externen Standpunkt aus einzelne individuelle Entscheidungen als fehlerhaft diagnostiziert, ist deutlich. Der Unterschied besteht vor allem in der Behauptung der liberalen Paternalisten, den Individuen nicht wirklich externe Präferenzen aufzwingen, sondern ihrem Eigeninteresse zum Durchbruch verhelfen zu wollen. Wie wir hier sehen, ist diese Behauptung jedoch wenig überzeugend.

Um herauszufinden, ob ein Entscheidungsdefekt vorliegt, könnte man einen Schritt von einer konkreten, kurzfristigen Entscheidungssituation zurücktreten 
und in einem Gedankenexperiment fragen, ob man selbst seine kurzfristigen Entscheidungen und die zugrunde liegenden kurzfristigen Präferenzen für gut oder vernünftig hält. Brennan und Lomasky (1983) diskutieren die Rolle von langfristig orientierten Meta-Präferenzen, die aus solchen Überlegungen folgen würden, im Zusammenhang mit meritorischen Gütern. Eines ihrer zentralen Argumente ist auch in der Diskussion um paternalistische Interventionen anwendbar: Es könnte sein, dass Äußerungen langfristiger Präferenzen vor allem expressiven Charakter haben. Sie werden als grobe Idealvorstellungen formuliert, ohne sich wirklich im Detail über die damit verbundenen Kosten klar zu sein. Es fällt leicht, am Silvesterabend den Neujahrsvorsatz zu fassen, im nächsten Jahr das Rauchen aufzugeben und Marathonform zu erreichen, gerade weil man die Folgen des Entzugs und die Qualen des regelmäßigen Trainings zunächst einmal ausblendet. Es ist dann aber überraschenderweise ganz anders als im paternalistischen Ansatz unterstellt; gerade die langfristigen Präferenzen sind ein Resultat wenig informierter Überlegungen in eine unbestimmte Zukunft hinein; die kurzfristige Neigung zum Genuss erscheint dagegen nun in einem vergleichsweise positiveren Licht als rationale Wahl.

Ein anderer Fall liegt vor, wenn die Individuen nur schwach ausgeprägte Präferenzen haben, dafür aber von den Umständen einer konkreten Entscheidungssituation stark in ihrer Entscheidung beeinflusst werden. Hier ist nochmals das schon oben erwähnte Ernährungsbeispiel illustrativ: Wird die ungesunde Eiscreme in den hintersten Ecken des Geschäfts versteckt, so werden Individuen mit schwachen Präferenzen, ohne viel darüber nachzudenken, die nahe liegenden Karotten auswählen. Der Konsum von Individuen mit nur schwachen, oder in einer konkreten Situation überhaupt nicht genau definierten Vorlieben wird also, für die Betroffenen kaum wahrnehmbar, durch die Präferenz des paternalistischen Planers für gesundes Essen gelenkt. Dieser orientiert sich seinerseits ziemlich eindimensional an einem Ziel, das schlicht dem common sense entspricht, aber keinem durchdachten, Opportunitätskosten in Rechnung stellenden, ökonomischen Wohlfahrtskalkül entspringt: an körperlicher Fitness. Dies mag zwar ein im öffentlichen Diskurs aktuell populäres Ziel sein, das Vorgehen an und für sich wirft jedoch eine Frage auf: Wenn ein pragmatisch am Zeitgeist orientierter liberaler Paternalist heute den Konsum vollwertiger Gesundheitskost propagiert, hätte er dann in den Jahren des Wirtschaftswunders zu einer besonders großen Portion Buttercremetorte geraten?

Die normative Basis des liberalen Paternalismus ist also denkbar schwach. Auch wenn man der Verhaltensökonomik folgt und die Existenz inkohärenter Präferenzen konstatiert, so bleibt es doch mehr als unklar, wie hiervon ausgehend ein paternalistisches Eingreifen tatsächlich zuverlässig im Sinne der Individuen gestaltet werden könnte. Die Orientierung an allgemeinen Zeitgeist-Phänomenen und Trends, wie es in der pragmatischen Variante des neuen Paternalismus geschieht, ist jedenfalls wenig überzeugend und wird auch dem normativen Individualismus, der eigentlich einem ökonomischen Ansatz zugrunde liegen sollte, in keiner Weise gerecht.

\subsection{Die politische Ökonomie des liberalen Paternalismus}

Die Ausführungen der Vertreter des liberalen Paternalismus sind häufig relativ vage, sobald es um die Kompetenzverteilung bei der Implementierung ihrer Konzepte 
geht. So werden in Thaler und Sunstein (2008) viele denkbare Anwendungsfälle zwar ausführlich diskutiert. Auch wird das aus Sicht der Autoren sinnvolle Design von Entscheidungssituationen im Detail erläutert. Die Frage, wer eigentlich mit der Umsetzung beauftragt werden soll, bleibt aber häufig offen. Ihr eigenes Programm Save More Tomorrow haben sich Thaler und Sunstein beispielsweise als Handelsmarke registrieren lassen. Es würde sich hier also um eine ganz normale Marktlösung zur Bereitstellung einer Selbstbindung handeln, so dass von Paternalismus eigentlich gar keine Rede sein kann. Das Beispiel der gesundheitsfördernden Anordnung von Lebensmitteln in einer Cafeteria dagegen kann als Appell an die Eigentümer einer solchen gelesen werden. Dass staatliches Eingreifen gefordert wird, falls der Appell ungehört bleibt, ist aber durchaus zu erwarten. Denn an anderer Stelle befürworten Thaler und Sunstein ausdrücklich staatlich gestützten Paternalismus. Dort geht es um regulierende Eingriffe zur Standardisierung von Informationen und auch Produkten mit dem Ziel, Preisvergleiche für Konsumenten zu erleichtern.

Sugden (2008) argumentiert ausführlich, dass solche staatlichen Interventionen auch zur Implementierung von nur liberalem und nicht hartem Paternalismus in Fällen wie dem Cafeteria-Beispiel zwingend notwendig sein werden, da beispielsweise eine Gewinn maximierende und eine Übergewicht minimierende Unternehmensführung nicht systematisch deckungsgleich sein werden. Darüber hinaus stellt Sugden aber auch die Bedeutung von Freiräumen für Kreativität im Marktprozess heraus. Gerade weil Unternehmer mit Neuerungen experimentieren, und auch weil Betreiber von Cafés so triviale Dingen wie das Angebot von Lebensmitteln und dessen Präsentation variieren, entdecken Konsumenten mit schwachen Präferenzen, dass ihnen Konsumgüter einen Nutzen stiften, von deren Existenz sie vorher vielleicht noch gar nichts wussten. Menschen profitieren von den Zufälligkeiten des Marktprozesses, gerade weil das verhaltensökonomische Argument zutrifft, dass sie keine vollständigen, konstanten und konsistenten Präferenzen haben. In einer dem statischen Denken liberaler Paternalisten entsprechend durchgestalteten Welt „optimaler" Entscheidungssituationen wäre diese Spontaneität aber drastisch reduziert.

Welche Art von Interventionen können wir nun erwarten, wenn die Politik sich des liberalen Paternalismus annimmt? Wie bereits oben gesehen, ist sich der neue Paternalismus seiner eigenen normativen Grundlagen nicht ganz sicher. Die zentrale Frage ist daher vor allem, welche Ziele sich eine neue paternalistische Politik voraussichtlich geben würde. Oben wurde bereits darauf hingewiesen, dass geäußerte langfristige Präferenzen häufig expressiven Charakter haben; man äußert Wunsch- und Idealvorstellungen, ohne sich über die in der Zukunft liegenden Kosten ihrer Realisierung ernsthafte Gedanken zu machen, oder sogar ohne die tatsächliche Realisierung wirklich ernsthaft ins Auge zu fassen.

Hillman (2010) formuliert eine allgemeine Theorie expressiven Verhaltens bei politischen Entscheidungen. Auf der individuellen Ebene liegt das Grundproblem expressiver Entscheidungen im Zusammenspiel von materiellem Eigeninteresse und dem Nutzen, den jemand aus dem Aufbau einer bestimmten, auch öffentlichen Identität für sich zieht. Auf der gesellschaftlichen Ebene kommt das Problem gegenseitiger Erwartungen hinzu, so dass expressiv Handelnde oft ihren Identitätsnutzen daraus ziehen, bei anderen Individuen keinen Anstoß zu erregen, oder im Idealfall sogar Wohlwollen hervorzurufen. Hillman nennt als Beispiel 
den Fall, dass es völlig rational sein kann, sich durch expressives Handeln ein pazifistisches Selbstbild zu erarbeiten, solange man davon ausgehen kann, dass die eigenen Äußerungen nicht gesellschaftlich entscheidend sind und die Existenz einer vorhandenen Verteidigung gegen äußere Bedrohungen nicht wirklich gefährden. Problematisch wird dies allerdings, wenn, wie in Kleinkostensituationen ${ }^{8}$ oft beobachtbar, individuell folgenloses Verhalten in der Summe vieler expressiver Handlungen dann doch gesellschaftlich bestimmend wird - dann kann sich leicht eine Politik durchsetzen, die mit den materiellen Eigeninteressen aller Beteiligten in Konflikt steht.

Man kann sich leicht vorstellen, wie sich solche Anreize auf die politische Festlegung von Zielen für paternalistische Interventionen auswirken werden. Eine individuelle Neigung, Langfristpräferenzen eher expressiv zu formulieren, wird hier gesellschaftlich nochmals verstärkt. Wenn nun ein liberaler Paternalismus ohne ein durchdachtes, operationalisierbares Wohlfahrtskonzept auskommen muss und sich stattdessen pragmatisch-willkürlich an zeitgeistigen Zielvorstellungen orientiert, dann ist zu erwarten, dass diese Politik selbst ineffizient ist. Denn sie gewichtet systematisch kurzfristige Kosten zu gering, expressiv formulierte Langfristpräferenzen aber zu hoch.

Man könnte nun argumentieren, dass dies alles kein Problem ist, da liberaler Paternalismus vorsieht, den Individuen die Möglichkeit zu einer Wahlentscheidung zu lassen, die sich vom Ziel des paternalistischen Planers unterscheidet. Hierzu sind zwei Punkte zu entgegnen: Erstens wohnt, wie oben gesehen, auch dem liberalen Paternalismus eine Tendenz zur Standardisierung inne. Wird er konsequent umgesetzt und ist er erfolgreich, dann werden Individuen mit eher schwachen Präferenzen in Richtung eines Normkonsums gelenkt und nur Individuen mit ohnehin schon relativ starken abweichenden Präferenzen nehmen auch bewusst die Kosten eines abweichenden Konsums auf sich. Eine möglicherweise große Gruppe von Individuen mit zunächst schwachen Präferenzen, die nicht der Vielfalt, den Zufällen und den Überraschungen nicht-paternalistisch gestalteter Entscheidungssituationen ausgesetzt ist, wird also möglicherweise nie durch Versuch und Irrtum erfahren, wie hoch die Opportunitätskosten des Normkonsums tatsächlich sind. Zweitens ist zu bedenken, dass ein politisch gestützter, zeitgeistiger Paternalismus gerade diesen Zeitgeist möglicherweise verstärkt und stabilisiert. Auch auf der gesellschaftlichen Ebene besteht also die Gefahr, dass das Lernen aus Versuch und Irrtum behindert und verlangsamt wird. ${ }^{9}$

\subsection{Paternalismus oder individuelles Problemlösen?}

Wie wir gesehen haben, besteht für Individuen, die sich ihrer Selbstkontrolldefizite, ihrer Entscheidungsschwächen und der Inkonsistenz ihrer Präferenzordnung bewusst sind, sehr häufig die Möglichkeit, selbst Mechanismen zur Lösung daraus entstehender Probleme zu finden. Hinzu kommt die Tatsache, dass die meisten der Anwendungsbeispiele, die liberale Paternalisten für ihr Konzept anführen, tatsächlich vor allem oder sogar ausschließlich intra-individuelle Probleme

8. Vgl. zum Konzept der Kleinkostensituationen Kirchgässner (1992).

9. Vgl. hierzu ausführlich Schnellenbach (2012). 
betreffen. Ein Selbstkontrolldefizit mag einen Konflikt zwischen dem heutigen und dem späteren Ich auslösen, aber es bleibt zunächst einmal offen, wo das gesellschaftliche Interesse an einer politischen Lösung für einen solchen Konflikt liegen könnte. Ein denkbarer Ansatzpunkt wären natürlich negative Externalitäten, etwa in Form höherer Kosten im Gesundheitswesen durch Rauchen oder ungesunde Ernährung. Hier legt die empirische Evidenz jedoch den Schluss nahe, dass bei einer Betrachtung über alle sozialen Sicherungssysteme hinweg solche negativen Externalitäten sehr klein sind, oder gar nicht existieren (etwa Viscusi 1999 sowie Bhattacharya und Sood 2011).

Nun sei für einen Moment - um des Argumentes willen und entgegen aller oben diskutierten Einwände - angenommen, dass eine klug gestaltete paternalistische Politik fehlerhafte individuelle Entscheidungen treffsicher korrigieren kann. Sollte man eine solche Politik befürworten? An dieser Stelle ist ein Blick auf die aktuelle Diskussion um politische Implikationen der Glücksforschung hilfreich, in der es um eine sehr ähnliche Fragestellung geht, nämlich inwieweit man durch ein politisches Glücks-Mikromanagement die Wohlfahrt der Individuen befördern kann und soll. Schubert (2012) weist in diesem Zusammenhang auf die in der ökonomischen Glücksliteratur eher vernachlässigte, in der psychologischen Literatur aber gut dokumentierte Tatsache hin, dass Autonomie und individuelle Handlungskompetenz eine wichtige Quelle von Lebenszufriedenheit sind. Nimmt man den Individuen durch paternalistische Steuerung die Notwendigkeit zur selbständigen Bewältigung von Problemen, die aus verhaltensökonomisch identifizierten Entscheidungsdefekten folgen, dann reduziert man damit in der längeren Frist aber auch ihre Chancen, aus eigener Erfahrung Handlungskompetenz zu entwickeln. Das paternalistisch behütete Individuum wird von der selbständigen Problemlösung als einer wichtigen Quelle von Wohlfahrt abgeschnitten und erlernt dadurch möglicherweise auch gar nicht mehr die Fähigkeiten, die hierzu nötig wären.

Berücksichtigt man dies, so wird man gegenüber einer paternalistischen Politik selbst dann skeptisch sein, wenn sie demokratisch, oder gar direktdemokratisch, legitimiert ist. Der Einwand besteht eben nicht nur darin, dass, wie im vorangegangenen Abschnitt bereits diskutiert, die üblichen Themen paternalistischer Politik besonders anfällig sind für die Fallen expressiver Politik (Glaeser 2006). Zumindest ebenso schwer wiegt auch der Einwand, dass hier ein Eingriff in die Privatautonomie mit, wie gesehen, potentiell erheblichen nicht intendierten Nebenwirkungen erfolgen würde, dem aber keinerlei gesellschaftlicher Handlungsbedarf zugrunde liegt, wie es etwa bei einem bedeutsamen Externalitätenproblem der Fall wäre.

Hinzu kommt schließlich auch noch ein dritter Einwand: Paternalistische Politik hat immer auch Verteilungsimplikationen. Heterogenität der Individuen in ihren Präferenzen und auch ihren Neigungen $\mathrm{zu}$ Entscheidungsdefekten vorausgesetzt, wird durch eine einheitliche paternalistische Politik Wohlfahrt umverteilt. Minderheiten werden durch eine für sie schädliche, jedenfalls aber unerwünschte paternalistische Politik belastet. Während also fehlerhafte Entscheidungen einzelner Individuen eine im Großen und Ganzen private Angelegenheit ohne nennenswerte externe Effekte sind, werden durch eine paternalistische Politik erst Kosten einer - möglicherweise - für einzelne Individuen erreichbaren Verbesserung ihrer Entscheidungsqualität auf andere Gruppen externalisiert. 


\section{Fazit}

Das Konzept des liberalen Paternalismus kann aus einer Reihe von Gründen nicht als sinnvolle Grundlage für politische Interventionen angesehen werden, und zwar selbst dann, wenn man die Resultate der jüngeren Verhaltensökonomik zustimmend zur Kenntnis nimmt und akzeptiert, dass Menschen nicht durch eine vollständige, konstante und widerspruchsfreie Präferenzordnung charakterisiert werden können. Die Diskussion in diesem Papier hat im Gegenteil gezeigt, dass liberaler Paternalismus ein zweifelhaftes Konzept ist, gerade wenn man verhaltensökonomische Erkenntnisse konsequent berücksichtigt. Dem liberalen Paternalismus fehlt eine überzeugende normative Grundlage.

Auch eine Orientierung an von Individuen selbst geäußerten Langfristpräferenzen stellt hier keine überzeugende Lösung dar, da diese häufig selbst verzerrt sind und die kurzfristigen Kosten der langfristigen Zielerreichung unterschätzen. Darüber hinaus spricht gerade die Nicht-Vollständigkeit von individuellen Präferenzordnungen dafür, die Spontaneität und Offenheit des Marktprozesses nicht durch paternalistisches Design von Entscheidungssituationen zu untergraben. Vor diesem Hintergrund ist es zwar denkbar, dass in Einzelfällen der Übergang von hartem zu liberalem Paternalismus einen kleinen Forschritt darstellen würde. ${ }^{10}$ Als allgemeine theoretische Rechtfertigung zur punktuellen Intervention in Marktprozesse erweist sich der liberale Paternalismus jedoch als untauglich.

\section{Literaturverzeichnis}

Akerlof, G.A. und W.T. Dickens (1982), The Economic Consequences of Cognitive Dissonance, American Economic Review 72, 307-319.

Bernheim, B.D. und A. Rangel (2004), Addiction and Cue-Triggered Decision Processes, American Economic Review 94, 1558-1590.

Bernheim, B.D. und A. Rangel (2007), Behavioral Public Economics: Welfare and Policy Analysis with Nonstandard Decision-Makers, in: P. Diamond und H. Vartiainen (eds.), Behavioral Economics and Its Applications. Princeton University Press, Princeton.

Besley, T. (1988), A Simple Model for Merit Good Arguments, Journal of Public Economics 35, 371-383.

Bhattacharya, J. und N. Sood (2011), Who Pays for Obesity?, Journal of Economic Perspectives 25 (1), 139-158.

Brennan, G.B. und L. Lomasky (1983), Institutional Aspects of 'Merit Goods' Analysis, Finanzarchiv N.F. 41, 183-206.

Brunello, G., P.-C. Michaud und A. Sanz-de-Galdeano (2009), The Rise of Obesity in Europe: An Economic Perspective, Economic Policy 59, 551-584.

Camerer, C.F., S. Issacharoff, G. Loewenstein, T. O’Donoghue und M. Rabin (2003), Regulation for Concervatives: Behavioral Economics and the Case for 'Asymmetric Paternalism', University of Pennsylvania Law Review 151, 1211-1254.

Choi, J.J., D. Laibson, C. Madian und A. Metrick (2006), Saving for Retirement on the Path of Least Resistance, in: E.J. McCaffrey und J. Slemrod (eds.), Behavioral Public Finance: Towards a New Agenda. Russell Sage Foundation, New York.

10. So zeigt beispielsweise Schwarz (2009), dass der Übergang vom traditionellen zum liberalen Paternalismus in der Regulierung des Tabakkonsums durchaus ein rationaler Schritt wäre. 


\section{Jan Schnellenbach}

Cordes, C. und C. Schubert (2011), Role Models that Make You Unhappy: Light Paternalism, Social Learning and Welfare, Papers on Economics and Evolution \#1022. Max-Planck-Institut für Ökonomik, Jena.

DellaVigna, S. (2009), Psychology and Economics: Evidence from the Field, Journal of Economic Literature 47, 315-372.

Dillis, A.K. und J.A. Miron (2004), Alcohol Prohibition and Cirrhosis, American Law and Economics Review 6, 285-318.

Glaeser, E.L. (2006), Paternalism and Psychology, University of Chicago Law Review 73, 133156.

Gneezy, U. und A. Rustichini (2000), A Fine is a Price, Journal of Legal Studies 29, 1-17.

Gruber, J. und B. Köszegi (2004), A Theory of Government Regulation of Addictive Bads: Tax Levels and Tax Incidence for Cigarette Excise Taxation, Journal of Public Economics 88, 1959-1987.

Gul, F. und W. Pesendorfer (2001), Temptation and Self-Control, Econometrica 69, 14031435.

Heinemann, F. (2010), Ansatzpunkte einer Gewissensökonomik, ORDO Jahrbuch für die Ordnung von Wirtschaft und Gesellschaft 61, 151-167.

Hillman, A.L. (2010), Expressive Behavior in Economics and Politics, European Journal of Political Economy 26, 403-418.

Kaur, S., M. Kremer und S. Mullainathan (2010), Self-Control and the Development of Work Arrangements, American Economic Review (P\&P) 100, 624-628.

Kirchgässner, G. (1992), Toward a Theory of Low-Cost Decisions, European Journal of Political Economy 8, 305-320.

Laibson, D. (1997), Golden Eggs and Hyperbolic Discounting, Quarterly Journal of Economics 112, 443-477.

Loewenstein, G. und P.A. Ubel (2008), Hedonic Adaptation and the Role of Decision and Experience Utility in Public Policy, Journal of Public Economics 92, 1795-1810.

Pazner, E.A. (1972), Merit wants and the theory of taxation, Public Finance/Finances Publiques $27,460-471$.

Schnellenbach, J. (2012), Nudges and Norms: On the Political Economy of Soft Paternalism, European Journal of Political Economy 28, 266-277.

Schubert, C. (2012), Pursuing Happiness, Kyklos 65, 245-261.

Schwarz, P. (2009), Optionen einer rationalen Regulierung des Tabakkonsums: Die Vorteile eines liberalen Paternalismus, Perspektiven der Wirtschaftspolitik 10, 235-251.

Sugden, R. (2008), Why Incoherent Preferences Do Not Justify Paternalism, Constitutional Political Economy 19, 226-248.

Sunstein, C.R. und R.H. Thaler (2003), Libertarian Paternalism, American Economic Review (P\&P) 93, 175-179.

Sunstein, C.R. und R.H. Thaler (2003a), Libertarian Paternalism Is Not An Oxymoron, University of Chicago Law Review 70, 1159-1202.

Thaler, R.H. und S. Benartzi (2004), Save More Tomorrow: Using Behavioral Economics to Increase Employee Saving, Journal of Political Economy 112 (Supplement), 164187.

Thaler, R.H. und C.R. Sunstein (2008), Nudge: Improving Decisions on Health, Wealth and Happiness. Yale University Press, New Haven.

Tversky, A. und D. Kahneman (1981), The Framing of Decisons and the Psychology of Choice, Science 211: 453-458.

Viscusi, W.K. (1999), The Governmental Composition of the Insurance Cost of Smoking, Journal of Law and Economics 42, 575-609.

Wohlgemuth, M. (2011), Hayek: ein klassisch-liberaler Paternalist? in: G. Schwarz und M. Wohlgemuth (Hrsg.), Das Ringen um die Freiheit. Die Verfassung der Freiheit nach 50 Jahren. NZZ libro, Zürich. 
Abstract: We discuss the idea of libertarian paternalism, which supposedly allows individuals to make better decisions, if they suffer from typical shortcomings in individual rationality, as they have been identified by behavioral economics. In particular, we discuss the normative foundations of libertarian paternalism, and decision-making on paternalist policies in a political economy framework. It is argued that a systematic improvement of the quality of individual decision-making through paternalist policies is not to be expected, and that serious unintended consequences may occur. 